\title{
PAR-Aware Multi-User Precoder for the Large-Scale MIMO-OFDM Downlink
}

\author{
Christoph Studer* and Erik G. Larsson ${ }^{\ddagger}$ \\ * Rice University, Houston, TX, USA; e-mail: studer@ rice.edu \\ †Linköping University, Linköping, Sweden; e-mail: erik.larsson@isy.liu.se
}

\begin{abstract}
We consider an orthogonal frequency-division multiplexing (OFDM)-based downlink transmission scheme for largescale multi-user (MU) multiple-input multiple-output (MIMO) wireless systems. In order to transmit signals with low peak-toaverage (power) ratio (PAR), we propose to exploit the massive degrees-of-freedom available in large-scale MU-MIMO-OFDM systems. Specifically, we jointly perform MU precoding, OFDM modulation, and PAR reduction by solving a convex optimization problem at the base station. Numerical results demonstrate tremendous PAR-reduction capabilities of the proposed method, which eventually enables us to use low-cost RF components for the large-scale MU-MIMO-OFDM downlink.
\end{abstract}

\section{INTRODUCTION}

Large-scale multiple-input multiple-output (MIMO) wireless communication systems are a promising means to meet the growing demands for higher throughput and improved quality-of-service of next-generation multi-user (MU) wireless systems [2]. The vision is that a large number of antennas at the base-station (BS) serve a large number of users concurrently and in the same frequency band, but with the number of BS antennas being much larger than the number of users [3], say a hundred antennas serving ten users. Large-scale MIMO systems also have the potential to reduce the operational power consumption at the transmitter and enable the use of lowcomplexity schemes for suppressing MU interference (MUI). All these properties render large-scale MIMO a promising technology for next-generation wireless systems.

While the theoretical aspects of large-scale MU-MIMO systems have gained significant attention in the research community (see, e.g., [2] for an overview), much less is known about practical transmission schemes. As it was realized in [4], practical implementations of large-scale MIMO systems will require the use of low-cost and low-power radio-frequency (RF) components. To this end, reference [4] proposed a MU precoding scheme for frequency-flat channels,

An extended version of this paper will appear in the IEEE Journal on Selected Areas in Communications [1].

The work of C. Studer was supported by the Swiss National Science Foundation (SNSF) under Grant PA00P2-134155. The work of E. G. Larsson was supported by the Swedish Foundation for Strategic Research (SSF), the Swedish Research Council (VR), and ELLIIT. E. G. Larsson is a Royal Swedish Academy of Sciences (KVA) Research Fellow supported by a grant from the Knut and Alice Wallenberg Foundation.

The authors would like to thank R. G. Baraniuk, E. Karipidis, A. Maleki, Saif K. Mohammed, and A. C. Sankaranarayanan for inspiring discussions. which relies on per-antenna constant-envelope (CE) transmission to enable efficient implementation using non-linear RF components.

Practical wireless channels typically exhibit frequencyselective fading and a low peak-to-average (power) ratio (PAR) precoding solution suitable for such channels would be desirable. Preferably, the solution should be such that the complexity required in each (mobile) terminal is small (due to stringent area and power constraints), whereas heavier processing could be afforded at the BS. Orthogonal frequency-division multiplexing (OFDM) [5] is an attractive and well-established way of dealing with frequency-selective channels. In addition to simplifying the equalization at the receiver, OFDM also facilitates per-tone power and bit allocation, scheduling in the frequency domain, and spectrum shaping. However, OFDM is known to suffer from a high PAR, which necessitates the use of linear RF components (e.g., power amplifiers) to avoid out-of-band radiation and signal distortions. Unfortunately, linear RF components are, in general, more costly and less power efficient than their non-linear counterparts, which would eventually result in exorbitant costs for large-scale BS implementations having hundreds of antennas. Therefore, it is of paramount importance to reduce the PAR of OFDM-based large-scale MU-MIMO systems to facilitate corresponding low-cost and low-power BS implementations.

To combat the challenging linearity requirements of OFDM, a plethora of PAR-reduction schemes have been proposed for point-to-point single-antenna and MIMO wireless systems (see, e.g., [6] for an overview). For MU-MIMO systems, however, a straightforward adaptation of these schemes is nontrivial, mainly because MU systems additionally require the removal of MUI using a precoder [7]. PAR-reduction schemes suitable for the MU-MISO and MU-MIMO downlink were described in [8] and [9], respectively, and rely on TomlinsonHarashima precoding. Both schemes, however, require specialized signal processing in the (mobile) terminals (e.g., modulo reduction), which prevents their use in conventional MIMOOFDM systems, such as IEEE $802.11 \mathrm{n}$ or 3GPP LTE.

\section{A. Contributions}

In this paper, we present a novel downlink transmission scheme for large-scale MU-MIMO-OFDM wireless systems. The proposed method only affects the signal processing at 
the BS while leaving the processing required at each terminal untouched. The key idea is to exploit the excess of degreesof-freedom (DoF) offered by equipping the BS with a large number of antennas and to jointly perform MU precoding, OFDM modulation, and PAR reduction, referred to as PMP in the remainder of the paper. We formulate PMP as a convex optimization problem and demonstrate its PAR-reduction capabilities using numerical simulations.

\section{B. Notation}

Lowercase boldface letters stand for column vectors and uppercase boldface letters designate matrices. For a matrix $\mathbf{A}$, we denote its transpose, conjugate transpose, and pseudoinverse by $\mathbf{A}^{T}, \mathbf{A}^{H}$, and $\mathbf{A}^{\dagger}=\mathbf{A}^{H}\left(\mathbf{A} \mathbf{A}^{H}\right)^{-1}$, respectively. The entry in the $k$ th row and $\ell$ th column of $\mathbf{A}$ is $[\mathbf{A}]_{k, \ell}$; the $k$ th entry of $\mathbf{a}$ is designated by $[\mathbf{a}]_{k}$. The $M \times M$ identity matrix is denoted by $\mathbf{I}_{M}$, the $M \times N$ all-zeros matrix by $\mathbf{0}_{M \times N}$, and $\mathbf{F}_{M}$ refers to the $M \times M$ discrete Fourier transform (DFT) matrix. The Euclidean (or $\ell_{2}$ ) norm of $\mathbf{a}$ is denoted by $\|\mathbf{a}\|_{2}$, $\|\mathbf{a}\|_{\infty}=\max _{k}\left|[\mathbf{a}]_{k}\right|$ stands for the $\ell_{\infty}$-norm, and the $\ell_{\widetilde{\infty}^{-}}$ norm [10] is defined as $\|\mathbf{a}\|_{\widetilde{\infty}}=\max \left\{\|\Re\{\mathbf{a}\}\|_{\infty},\|\Im\{\mathbf{a}\}\|_{\infty}\right\}$ with $\Re\{\mathbf{a}\}$ and $\Im\{\mathbf{a}\}$ representing the real and imaginary part of $\mathbf{a}$, respectively. Sets are designated by upper-case calligraphic letters; the cardinality and complement of the set $\mathcal{T}$ is $|\mathcal{T}|$ and $\mathcal{T}^{c}$, respectively.

\section{PRELIMINARIES}

We start by introducing the system model followed by an overview of MU precoding schemes and of fundamental PAR issues arising in OFDM-based communication systems.

\section{A. System Model}

We consider an OFDM-based MU-MIMO downlink scenario as depicted in Fig. 1. The BS is assumed to have a significantly larger number of transmit antennas $N$ than the number $M \ll N$ of independent terminals (users); each terminal is equipped with a single antenna only. The signal vector $\mathbf{s}_{w} \in \mathcal{O}^{M}$ contains information for each of the $M$ users, where $w=1, \ldots, W$ indexes the OFDM tones, $W$ corresponds to the total number of OFDM tones, $\mathcal{O}$ represents the set of scalar complex-valued constellations, and $\left[\mathbf{s}_{w}\right]_{m} \in \mathcal{O}$ corresponds to the symbol at tone $w$ to be transmitted to user $m$. We normalize the symbols to satisfy $\mathbb{E}\left\{\left|\left[\mathbf{s}_{w}\right]_{m}\right|^{2}\right\}=1 / M$. To shape the spectrum of the transmitted signals, OFDM systems typically specify certain unused tones (e.g., at both ends of the spectrum [5]). Hence, we set $\mathbf{s}_{w}=\mathbf{0}_{M \times 1}$ for $w \in \mathcal{T}^{c}$ where $\mathcal{T}$ designates the set of tones used for data transmission.

In order to remove MUI, the signal vectors $\mathbf{s}_{w}, \forall w$ are passed through a precoder, which generates $W$ vectors $\mathbf{x}_{w} \in \mathbb{C}^{N}$ according to a given precoding scheme (see Section II-B). Since precoding causes the transmit power $P=\sum_{w=1}^{W}\left\|\mathbf{x}_{w}\right\|_{2}^{2}$ to depend on the signals $\mathbf{s}_{w}, \forall w$ and the channel state, we normalize the precoded vectors $\mathbf{x}_{w}, \forall w$ prior to transmission as

$$
\hat{\mathbf{x}}_{w}=\mathbf{x}_{w} / \sqrt{\sum_{w=1}^{W}\left\|\mathbf{x}_{w}\right\|_{2}^{2}}, w=1, \ldots, W,
$$

to ensure unit transmit power. We emphasize that this normalization is an essential step in practice (i.e., to meet regulatory power constraints), but to simplify the presentation, the normalization is omitted in the description of the precoders to follow (but normalization employed in the simulation results shown in Section IV). Hence, in what follows $\mathbf{x}_{w}$ and $\hat{\mathbf{x}}_{w}$ are treated interchangeably.

The (normalized) vectors $\mathbf{x}_{w}, \forall w$ are then re-ordered (from user orientation to transmit-antenna orientation) according to the following one-to-one mapping:

$$
\left[\mathbf{x}_{1} \cdots \mathbf{x}_{W}\right]=\left[\mathbf{a}_{1} \cdots \mathbf{a}_{N}\right]^{T},
$$

where the $W$-dimensional vector $\mathbf{a}_{n}$ corresponds to the (frequency-domain) signal to be transmitted from the $n$th antenna. The time-domain samples are obtained by applying the inverse DFT (IDFT) according to $\hat{\mathbf{a}}_{n}=\mathbf{F}_{W}^{H} \mathbf{a}_{n}$ followed by parallel-to-serial (P/S) conversion. Prior to modulation and transmission over the wireless channel, a cyclic prefix (CP) is added to the (time-domain) samples $\hat{\mathbf{a}}_{n}, \forall n$ to avoid ISI [5].

To simplify the exposition, we specify the input-output relation of the wireless channel in the frequency domain only. Concretely, we consider

$$
\mathbf{y}_{w}=\mathbf{H}_{w} \mathbf{x}_{w}+\mathbf{n}_{w}, \quad w=1, \ldots, W,
$$

where $\mathbf{y}_{w}$ denotes the $w$ th receive vector, $\mathbf{H}_{w} \in \mathbb{C}^{M \times N}$ represents the MIMO channel matrix associated with the $w$ th OFDM tone, and $\mathbf{n}_{w}$ is an $M$-vector of i.i.d. complex Gaussian noise with zero-mean and variance $N_{0}$ per entry. The average receive signal-to-noise-ratio (SNR) is defined by $\mathrm{SNR}=1 / N_{0}$. Finally, each of the $M$ user terminals performs OFDM demodulation to obtain the received (frequencydomain) signals $\left[\mathbf{y}_{w}\right]_{m}, w=1, \ldots, W$ (see Fig. 1).

\section{B. Linear MU Precoding Schemes}

In order to avoid MUI, precoding must be employed at the BS. To this end, we assume the channel matrices $\mathbf{H}_{w}, \forall w$ to be known perfectly at the transmit-side. ${ }^{1}$ Linear precoding now amounts to transmitting $\mathbf{x}_{w}=\mathbf{G}_{w} \mathbf{s}_{w}$, where $\mathbf{G}_{w} \in \mathbb{C}^{N \times M}$ is a suitable precoding matrix. One of the most prominent precoding schemes is least-squares (LS) precoding (or linear zero-forcing precoding), which corresponds to $\mathbf{G}_{w}=\mathbf{H}_{w}^{\dagger}$ and perfectly removes all MUI since $\mathbf{H}_{w} \mathbf{H}_{w}^{\dagger}=\mathbf{I}_{M}$. In Section IV, we will also consider matched filter (MF) precoding, which amounts to setting $\mathbf{G}_{w}=\mathbf{H}_{w}^{H}$ but does not perfectly remove MUI. Nevertheless, MF precoding was shown in [3] to perfectly remove MUI in the large-antenna limit, i.e., when $N \rightarrow \infty$.

\section{Peak-to-Average Ratio (PAR)}

OFDM transmission causes the signals $\hat{\mathbf{a}}_{n}, \forall n$ to exhibit a large dynamic range [5]. Such signals are susceptible to non-linear distortions (e.g., saturation or clipping) typically induced by real-world RF components. To avoid unwanted

\footnotetext{
${ }^{1}$ In large-scale MU-MIMO systems, channel-state information at the transmitter would probably be acquired through pilot-based training in the uplink and by exploiting reciprocity of the wireless channel [2], [3]
} 

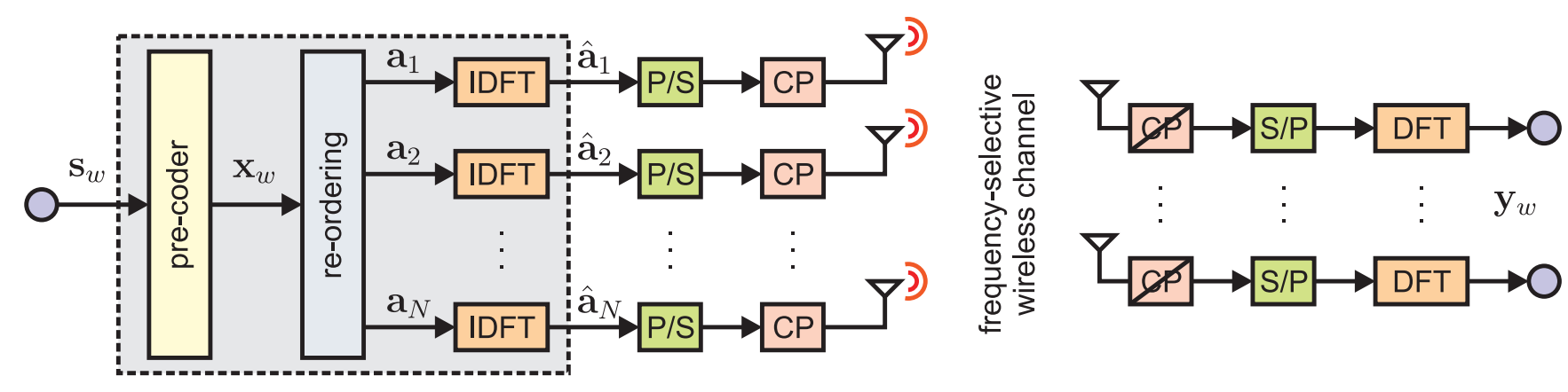

Fig. 1. Large-scale MU-MIMO-OFDM downlink (left: BS with $N$ transmit antennas; right: $M$ independent single-antenna terminals). The proposed downlink transmission scheme, referred to as PMP, combines MU precoding, OFDM modulation, and PAR reduction (highlighted by the dashed box in the BS).

out-of-band radiation and signal distortions altogether, linear $\mathrm{RF}$ components and PAR-reduction schemes are key to successfully deploy OFDM in practical systems.

1) PAR Definition: The dynamic range of the transmitted OFDM signals is typically characterized through the peak-toaverage (power) ratio (PAR). We define the PAR at the $n$th transmit antenna as ${ }^{2}$

$$
\operatorname{PAR}_{n}=\frac{2 W\left\|\hat{\mathbf{a}}_{n}\right\|_{\widetilde{\infty}}^{2}}{\left\|\hat{\mathbf{a}}_{n}\right\|_{2}^{2}} .
$$

To minimize distortion due to hardware non-linearities, the transmit signals should have a PAR that is close to one, which can either be achieved by CE transmission [4] or by using sophisticated PAR-reduction schemes.

2) PAR-Reduction Schemes for OFDM: In order to reduce the PAR in single-antenna and point-to-point MIMO systems, a large number of PAR-reduction algorithms have been developed in the last decade (see, e.g., [6] for an overview). For the MU-MIMO downlink, a method has been introduced recently in [9]; this method, however, requires dedicated signalprocessing algorithms at both ends of the wireless link (e.g., modulo reduction in the receiver). In contrast, the transmission scheme presented next aims at reducing the PAR by only exploiting the excess of transmit antennas available at the BS, which has the key advantage of being transparent to the receivers, i.e., it does not require any special signal-processing algorithms in the (mobile) terminals.

\section{Downlink Transmission Scheme}

The main idea of the downlink transmission scheme developed next is to jointly perform MU precoding, OFDM modulation, and PAR reduction, by exploiting the DoF available in large-scale MU-MIMO systems. To convey the underlying idea, we start by considering a simplified MIMO system. We then present the MU-MIMO-OFDM downlink transmission scheme in full detail.

\footnotetext{
${ }^{2}$ We emphasize that alternative PAR definitions exist in the literature, e.g., using the $\ell_{\infty}$-norm in the nominator instead of the $\ell_{\widetilde{\infty}}$-norm (and $W$ instead of $2 W$ ). Nevertheless, the relation $\frac{1}{2}\left\|\hat{\mathbf{a}}_{n}\right\|_{\infty}^{2} \leq\left\|\hat{\mathbf{a}}_{n}\right\|_{\widetilde{\infty}}^{2} \leq\left\|\hat{\mathbf{a}}_{n}\right\|_{\infty}^{2}$ shown in [10, Eq. 12] ensures that reducing the PAR as defined in (3) also reduces an $\ell_{\infty}$-norm-based PAR definition (and vice versa). Moreover, the theory and algorithms presented in this paper can, for example, be formulated to directly reduce an $\ell_{\infty}$-norm-based PAR.
}

\section{A. Basic Idea and Fundamental Properties}

Let us consider a narrow-band flat-channel MU-MIMO system with the real-valued input-output relation $\mathbf{y}=\mathbf{H x}+\mathbf{n}$ and an $M \times N$ channel matrix satisfying $M<N$. To eliminate MUI, the transmit-vector $\mathrm{x}$ must satisfy the precoding constraint $\mathbf{s}=\mathbf{H x}$, which ensures that $\mathbf{y}=\mathbf{s}+\mathbf{n}$ when transmitting the vector $\mathbf{x}$. Since $M<N$, the equation $\mathbf{s}=\mathbf{H x}$ is underdetermined; this implies that there are, in general, infinitely many solutions $\mathbf{x}$ satisfying the precoding constraint. In order to find a suitable vector $\dot{\mathrm{x}}$ having a small dynamic range (or low PAR), we consider the following convex optimization problem:

$$
\text { (P-INF) } \underset{\tilde{\mathbf{x}}}{\operatorname{minimize}}\|\tilde{\mathbf{x}}\|_{\infty} \quad \text { subject to } \mathbf{s}=\mathbf{H} \tilde{\mathbf{x}} \text {. }
$$

As (P-INF) minimizes the magnitude of the largest entry of $\tilde{\mathbf{x}}$, we can expect that its solution $\dot{x}$ exhibits low PAR.

To characterize the benefit of having a large number of transmit antennas at the BS on the PAR when using (P-INF) for precoding, we use a result derived in [11, Prop. 1]. If $\mathbf{H}$ has full rank and $1 \leq M<N$, then the PAR of the solution $\dot{\mathrm{x}}$ resulting from $(\mathrm{P}-\mathrm{INF})$ generally ${ }^{3}$ satisfies

$$
\operatorname{PAR}_{\text {P-INF }}=\frac{N\|\dot{\mathbf{x}}\|_{\infty}^{2}}{\|\dot{\mathbf{x}}\|_{2}^{2}} \leq \frac{N}{N-M+1} .
$$

From this observation, we see that for a constant number of users $M$ and in the large-antenna limit $N \rightarrow \infty$, the bound (4) implies that PAR $\mathrm{P}_{\mathrm{PINF}} \rightarrow 1$. Hence, for systems having a significantly larger number of transmit antennas than usersas is the case for typical large-scale MU-MIMO systems [2], [3] - a precoder that implements (P-INF) is able to achieve a PAR arbitrarily close to unity. This means that in the largeantenna limit of $N \rightarrow \infty$, (P-INF) yields constant-envelope signals, while being able to perfectly eliminate the MUI.

\section{B. Joint Precoding, Modulation, and PAR Reduction (PMP)}

The application of (P-INF) to each time-domain sample after OFDM modulation would reduce the PAR but, unfortunately, would no longer allow the equalization of ISI using conventional OFDM demodulation. To enable the use

\footnotetext{
${ }^{3}$ Note that [11, Prop. 1] implicitly excludes certain specific instances of $\mathbf{H}$.
} 
of conventional OFDM demodulation in the receiver, we next formulate the convex optimization problem to jointly perform MU precoding, OFDM modulation, and PAR reduction.

We start by specifying the necessary constraints. In order to remove MUI, the following precoding constraints must hold:

$$
\mathbf{s}_{w}=\mathbf{H}_{w} \mathbf{x}_{w}, w \in \mathcal{T}
$$

To ensure certain desirable spectral properties of the transmitted OFDM signals, the inactive OFDM tones (indexed by $\mathcal{T}^{c}$ ) must satisfy the following shaping constraints:

$$
\mathbf{0}_{N \times 1}=\mathbf{x}_{w}, w \in \mathcal{T}^{c} .
$$

PAR reduction is achieved similarly to (P-INF), with the main difference that we want to minimize the $\ell_{\widetilde{\infty}}$-norm of the time-domain samples $\hat{\mathbf{a}}_{n}, \forall n$. In order to simplify notation, we define the (linear) mapping between the time-domain samples $\hat{\mathbf{a}}_{n}, \forall n$, and the $w$ th (frequency-domain) transmit vector $\mathbf{x}_{w}$ as $\mathbf{x}_{w}=f_{w}\left(\hat{\mathbf{a}}_{1}, \ldots, \hat{\mathbf{a}}_{N}\right)$, where the linear function $f_{w}(\cdot)$ applies the DFT according to $\mathbf{a}_{n}=\mathbf{F}_{W} \hat{\mathbf{a}}_{n}, \forall n$ and performs the re-ordering defined in (1).

With (5) and (6), we are able to formulate the downlink transmission scheme as a convex optimization problem:

$$
(\text { PMP })\left\{\begin{array}{cl}
\operatorname{minimize}_{\tilde{\mathbf{a}}_{1}, \ldots, \tilde{\mathbf{a}}_{N}} & \max \left\{\left\|\tilde{\mathbf{a}}_{1}\right\|_{\widetilde{\infty}}, \ldots,\left\|\tilde{\mathbf{a}}_{N}\right\|_{\widetilde{\infty}}\right\} \\
\text { subject to } & \mathbf{s}_{w}=\mathbf{H}_{w} f_{w}\left(\tilde{\mathbf{a}}_{1}, \ldots, \tilde{\mathbf{a}}_{N}\right), w \in \mathcal{T} \\
& \mathbf{0}_{N \times 1}=f_{w}\left(\tilde{\mathbf{a}}_{1}, \ldots, \tilde{\mathbf{a}}_{N}\right), w \in \mathcal{T}^{c} .
\end{array}\right.
$$

The vectors $\hat{\mathbf{a}}_{n}, \forall n$ which minimize (PMP) correspond to the time-domain OFDM samples to be transmitted from each antenna. Following the reasoning of Section III-A, we expect these vectors to have low PAR (see Section IV for corresponding simulation results).

\section{Solving (PMP) at Low Computational Complexity}

The high dimensionality of (PMP) for large-scale MIMO systems necessitates corresponding efficient optimization algorithms. To this end, an efficient algorithm for solving a relaxed version of (PMP), referred to as fast iterative truncation algorithm (FITRA), was developed in [1]. This algorithm requires to restate (PMP) into Lagrangian form as

$$
\left(\text { PMP-L) } \underset{\overline{\mathbf{a}}}{\operatorname{minimize}} \lambda\|\overline{\mathbf{a}}\|_{\widetilde{\infty}}+\|\overline{\mathbf{b}}-\overline{\mathbf{C}} \overline{\mathbf{a}}\|_{2}^{2},\right.
$$

where $\overline{\mathbf{a}}=\left[\begin{array}{lll}\hat{\mathbf{a}}_{1}^{T} & \cdots & \hat{\mathbf{a}}_{N}^{T}\end{array}\right]^{T}$ contains all time-domain vectors, $\overline{\mathbf{b}}$ is a concatenation of $\mathbf{s}_{w}, w \in \mathcal{T}$ and $\left|\mathcal{T}^{c}\right|$ all-zeros vectors of dimension $N$, the matrix $\overline{\mathbf{C}}$ implements ${ }^{4}$ the right-hand-side of the constraints (5), and $\lambda>0$ is a regularization parameter.

A common approach to solve optimization problems of the form (PMP-L) is to use interior-point methods [12]. Such methods, however, often require prohibitively high computational complexity for the problem sizes faced in large-scale MIMO systems. Hence, to enable practical implementation of PMP, more efficient algorithms are of paramount importance. To this end, one can use FITRA developed in [1], which

\footnotetext{
${ }^{4}$ For the sake of simplicity of exposition, the actual structural details of the matrix $\overline{\mathbf{C}}$ are omitted
}

efficiently solves (PMP-L) and, hence, performs PMP at low computational complexity.

\section{Simulation Results}

In this section, we demonstrate the efficacy of the proposed joint precoding, modulation, and PAR reduction approach.

\section{A. Simulation Parameters}

The simulation results shown next are for a MU-MIMOOFDM system having $N=100$ antennas at the BS and serving $M=10$ single-antenna terminals. We employ OFDM with $W=128$ tones and use a spectral map $\mathcal{T}$ as specified in the $40 \mathrm{MHz}$-mode of IEEE $802.11 \mathrm{n}$. We consider coded transmission, i.e., for each user, we independently encode 216 information bits using a convolutional code (rate-1/2, generator polynomials $\left[133_{o} 171_{o}\right]$, and constraint length 7 ), apply random interleaving (across OFDM tones), and map the coded bits to a 16-QAM constellation (using Gray labeling).

To implement (PMP-L), we use FITRA as detailed in [1] with a maximum number of $K=2000$ iterations and a regularization parameter of $\lambda=0.25$. In addition to LS and MF precoding, we also consider a baseline precoding and PAR-reduction method. To this end, we employ LS precoding followed by truncation of the entries of the time-domain samples $\hat{\mathbf{a}}_{n}, \forall n$; we use a clipping strategy where one can specify a certain target PAR.

The precoded and normalized vectors are then transmitted over a frequency-selective channel modeled as a tap-delay line with $T=4$ taps. The time-domain channel matrices $\widehat{\mathbf{H}}_{t}$, $t=1, \ldots, T$, that constitute the impulse response of the channel, have i.i.d. circularly symmetric Gaussian distributed entries with zero mean and unit variance. To detect the transmitted information bits, each user $m$ performs soft-output demodulation of the received symbols $\left[\mathbf{y}_{w}\right]_{m}, w=1, \ldots, W$ and applies a soft-input Viterbi decoder.

\section{B. Performance Measures}

To compare the PAR characteristics of different precoding schemes, we use the complementary cumulative distribution function (CCDF) defined as

$$
\mathrm{CCDF}(\mathrm{PAR})=\mathbb{P}\left\{\mathrm{PAR}_{n}>\mathrm{PAR}\right\}
$$

The error-rate performance is characterized by the average (across users) symbol-error rate (SER); a symbol is said to be in error if at least one of the information bits per received OFDM symbol is decoded in error. To characterize the amount of signal power that is transmitted outside the set of active tones $\mathcal{T}$, we define the out-of-band (power) ratio (OBR) as follows:

$$
\mathrm{OBR}=\frac{|\mathcal{T}| \sum_{w \in \mathcal{T}^{c}}\left\|\mathbf{x}_{w}\right\|_{2}^{2}}{\left|\mathcal{T}^{c}\right| \sum_{w \in \mathcal{T}}\left\|\mathbf{x}_{w}\right\|_{2}^{2}}
$$

Note that for LS and MF precoding, we have OBR $=0$, as they operate independently on each of the $W$ tones; for PMP or LS followed by clipping, we have $\mathrm{OBR}>0$ in general. 


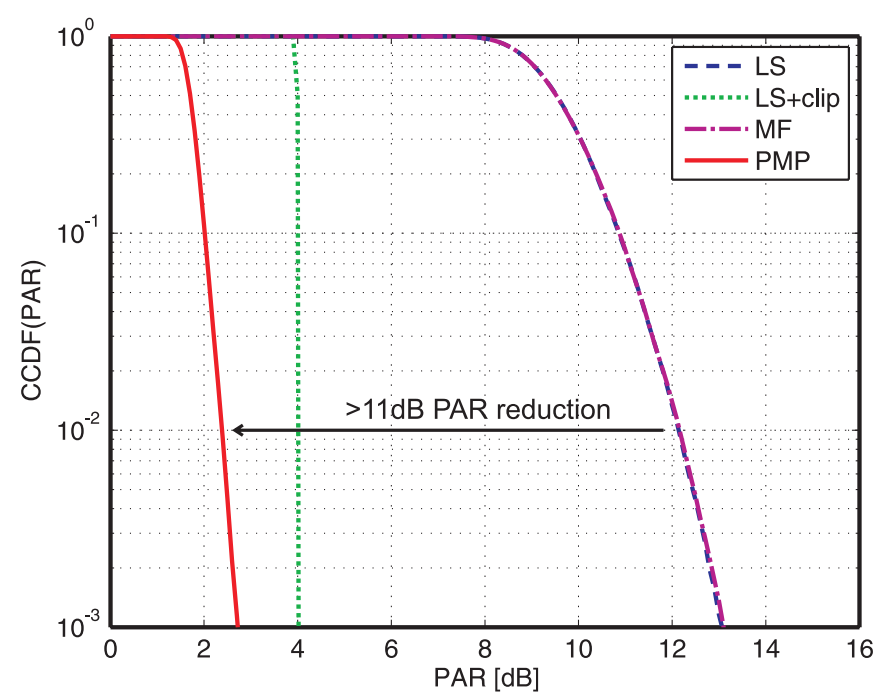

(a) PAR performance comparison. PMP effectively reduces the PAR compared to LS and MF precoding, whereas LS+clip achieves $4 \mathrm{~dB}$ PAR. (Note that the curves of LS and MF overlap.)

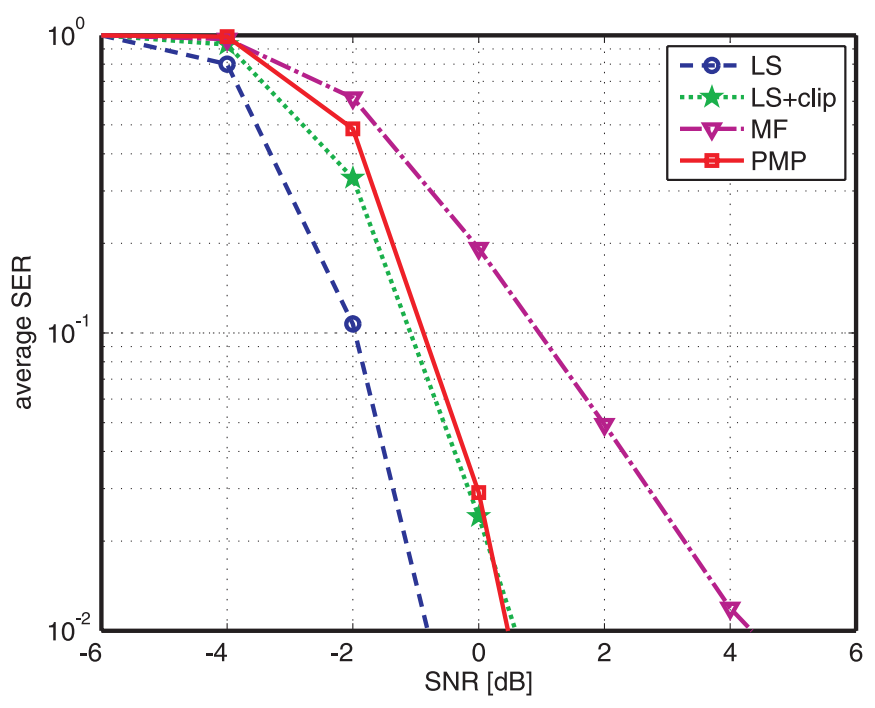

(b) Symbol error-rate (SER) performance. Renormalization causes $1 \mathrm{~dB}$ SNR-performance loss for PMP compared to LS. The loss of LS+clip is caused by normalization and residual MUI.

Fig. 2. PAR and SER performance for various precoding schemes. The target PAR for LS+clip is $4 \mathrm{~dB}$ and $\lambda=0.25$ for PMP using on FITRA.

\section{Performance and Capabilities of PMP}

Figure 2 demonstrates the efficacy of PMP and compares its PAR-reduction and error-rate performance to those of LS and MF precoding, as well as to LS precoding followed by clipping (denoted by "LS+clip"). One can immediately see that PMP reduces the PAR by more than $11 \mathrm{~dB}$ compared to LS and MF precoding (at CCDF $(\mathrm{PAR})=1 \%$ ); LS+clip achieves $4 \mathrm{~dB}$ PAR deterministically. In order to maintain a constant transmit power, the signals resulting from PMP require a stronger normalization (roughly $1 \mathrm{~dB}$ ) than the signals from LS precoding; this behavior causes the SNR-performance loss compared to LS (see Fig. 2(b)). The performance loss of MF and LS+clip is mainly caused by residual MUI. The OBR of LS+clip is $-11.9 \mathrm{~dB}$, which is a result of ignoring the spectral constraints, whereas the OBR of PMP is negligible (i.e., less than $-52 \mathrm{~dB}$ ). We finally emphasize that the regularization parameter $\lambda$ of FITRA used for solving (PMP) enables a trade-off between error-rate performance, OBR, and PARreduction performance. A corresponding detailed discussion can be found in the journal version of this paper [1].

\section{CONCLUSIONS}

We have proposed a framework to jointly perform precoding, modulation, and PAR reduction, referred to as PMP, which exploits the large number of degree-of-freedoms offered by large-scale MU-MIMO systems. In essence, the downlink channel matrix has a high-dimensional null-space, which enables us to shape the transmit signals to have "hardwarefriendly" properties, such as low PAR. By formulating PMP as a convex optimization problem, one can use the fast iterative truncation algorithm (FITRA) developed in [1] to carry out PMP at low computational complexity.

Numerical experiments have shown that PMP is able to reduce the PAR by more than $11 \mathrm{~dB}$ compared to conventional precoding methods, without creating noticeable out-of-band interference; both properties substantially alleviate the linearity requirements of the radio-frequency (RF) components. Furthermore, since PMP only affects the signal processing at the $\mathrm{BS}$, it can be deployed in existing MIMO-OFDM wireless communication systems, such as IEEE $802.11 \mathrm{n}$.

\section{REFERENCES}

[1] C. Studer and E. G. Larsson, "PAR-aware large-scale multi-user MIMOOFDM downlink," to appear in IEEE Journal on Selected Areas in Communications, preprint available: arXiv:1202.4034.

[2] F. Rusek, D. Persson, B. K. Lau, E. G. Larsson, O. Edfors, F. Tufvesson, and T. L. Marzetta, "Scaling up MIMO: opportunities and challenges with very large arrays," arXiv:1201.3210v1, Jan. 2012.

[3] T. L. Marzetta, "Non-cooperative cellular wireless with unlimited numbers of base station antennas," IEEE Trans. Wireless Comm., vol. 9, no. 11, pp. 3590-3600, Nov. 2010.

[4] S. K. Mohammed and E. G. Larsson, "Per-antenna constant envelope precoding for large multi-user MIMO systems," arXiv:1111.3752v1, Jan. 2012.

[5] R. van Nee and R. Prasad, OFDM for wireless multimedia communications. Artech House Publ., 2000.

[6] S. H. Han and J. H. Lee, "An overview of peak-to-average power ratio reduction techniques for multicarrier transmission," IEEE Wireless Comm., vol. 12, no. 2, pp. 1536-1284, Apr. 2005.

[7] R. F. H. Fischer, Precoding and Signal Shaping for Digital Transmission. Wiley, New York, 2002.

[8] S. K. Mohammed, A. Chockalingam, and B. S. Rajan, "A lowcomplexity precoder for large multiuser MISO systems," in IEEE Vehicular Tech. Conf (VTC), vol. Spring, Marina Bay, Singapore, May 2008, pp. 797-801.

[9] C. Siegl and R. F. H. Fischer, "Selected basis for PAR reduction in multi-user downlink scenarios using lattice-reduction-aided precoding," EURASIP J. on Advanced Sig. Proc., vol. 17, pp. 1-11, July 2011.

[10] D. Seethaler and H. Bölcskei, "Performance and complexity analysis of infinity-norm sphere-decoding," IEEE Trans. Inf. Th., vol. 56, no. 3, pp. 1085-1105, Mar. 2010.

[11] J.-J. Fuchs, "Spread representations," in Proc. 45th Asilomar Conf. on Signals, Systems, and Comput., Pacific Grove, CA, USA, 2011.

[12] S. Boyd and L. Vandenberghe, Convex Optimization. New York, NY, USA: Cambridge Univ. Press, 2004. 